\title{
Spectral Element Methods on Unstructured Meshes: Comparisons and Recent Advances
}

\author{
Richard Pasquetti ${ }^{1}$ and Francesca Rapetti ${ }^{1}$
}

Received October 5, 2004; accepted (in revised form) April 15, 2005; Published online March 8, 2006

\begin{abstract}
Spectral element approximations for triangles are not yet as mature as for quadrilaterals. Here we compare different algorithms and show that using an integration rule based on Gauss-points for simplices is of interest. We point out that this can be handled efficiently and allows to recover the convergence rate theoretically expected, even with curved elements.
\end{abstract}

KEY WORDS: spectral elements; triangular and quadrangular mesh elements; curved elements; Fekete points; Gauss-Lobatto points.

\section{INTRODUCTION}

The celebrated Spectral Element Method (SEM) has appeared to be of great interest to extend the capabilities of spectral methods to complex geometries. However, using quadrangular (hexahedral in 3D) elements may be a severe restriction for the most complex geometries, because requiring a structured mesh. Thus, Finite element meshes are usually based on triangles (tetrahedra in 3D). Efforts have been made in the late 1990s to implement spectral methods based on simplices. In [11] (and related works) it is proposed to use a "collapsed coordinate system", so that the integration rules for quadrangles may be used for triangles. Such an approach follows ideas developed earlier in the context of integration rules for simplices (see, e.g., [13] and references herein). Its main drawback is that the quadrature points are not symmetrically located in the triangle (tetrahedron), and a priori useless accumulations of quadrature points, also used as approximation points [11], arise in some vertices.

\footnotetext{
${ }^{1}$ Laboratory J.-A. Dieudonné, UMR CNRS 6621 and University de Nice-Sophia Antipolis, Parc Valrose, 06108 Nice Cedex 02, France. E-mails: \{rpas, frapetti\}@math.unice.fr
} 
Here we start from the triangle based SEM (say the TSEM) introduced in [16], where the Fekete points of the triangle are used as approximation points (see, e.g., $[3,8]$ in $2 \mathrm{D}$ and $[4,9]$ in $3 \mathrm{D}$ for other sets of points). This approach makes use of a nodal basis, i.e., Lagrange polynomials built on Fekete points are used as basis functions to span the space $\mathcal{P}_{N}(T)$ of all polynomials, defined on the triangle $T$, of total degree less or equal than $N$. Fekete points show some nice properties that are described in [15], where a computational algorithm and the location of the points are given for polynomial approximations of degree up to $N=18$. The Fekete points based TSEM was, e.g., used in [19], where an integration rule different from the one used in [16] was introduced. In [12], we have revisited the TSEM and also mentioned the importance of the integration rule.

In this paper, we focus on this latter point. To this end we consider elliptic problems, for which the exact solutions are known, and compare different approaches. The superiority of an approach based on Gauss points to compute the integrals is numerically proved, especially by showing that it supports the case of curved triangles with negligible additional computational cost. Moreover, we emphasize that a rather small number of quadrature points may allow to preserve the exponential convergence rate. In Sec. 2, we describe the different algorithms. In Sec. 3, we give comparisons for only one, non-curved and then curved spectral element, before providing a convergence study for a test-case involving the spectral element mesh of a non-polygonal domain.

\section{TSEM ALGORITHMS}

In this section, we describe the four following different approaches:

- TSEM-1: The Fekete point based method proposed in [16].

- TSEM-2: The Fekete point based method but improved by using the semi-analytical integration rule introduced in [19].

- TSEM-3: The Fekete point based method but using a Gauss points based integration rule.

- TSEM-4: The TSEM-3 with mass lumping.

TSEM-1: For the TSEM introduced in [16] the so-called Fekete points are used as approximation points and the Lagrange polynomials built on these points, say $\left\{\phi_{i}\right\}_{i=1}^{n}$, as basis functions. Let $\left\{\psi_{k}\right\}_{k=1}^{n}$, be an orthonormal basis of $\mathcal{P}_{N}(T)$, the space of polynomials defined on the triangle $T=\{(r, s),-1 \leqslant r, s \leqslant 1, r+s \leqslant 0\}$ and of total degree $\leqslant N$, for the usual $L^{2}(T)$ inner product (the Koornwinder-Dubiner (KD) polynomials 
may be used to constitute such a basis [7]). Then, Fekete points, say $\left\{\boldsymbol{x}_{i}\right\}_{i=1}^{n}$, are those which maximize the determinant of the Vandermonde matrix $V$, such that $V_{i j}=\psi_{j}\left(\boldsymbol{x}_{i}\right), 1 \leqslant i, j \leqslant n$, where $n=(N+1)(N+2) / 2$. Important properties of Fekete points are listed/proved in [15]. The main one is that Fekete points are Gauss-Lobatto points for the cube [1, 2], thus providing a strong link with the usual SEM. To set up the mass and stiffness matrices, in [16] it is suggested to use an integration rule that writes $I=\int_{\Omega} u v d \Omega \approx \sum_{i=1}^{n} u_{i} v_{i} J\left(\boldsymbol{x}_{i}\right) w_{i}$, for the product of functions $u$ and $v$ over the integration domain $\Omega$. Here $w_{i}$ are the quadrature weights, $J\left(\boldsymbol{x}_{i}\right)$ is the Jacobian determinant of the mapping $\boldsymbol{g}: T \rightarrow \Omega$ at the Fekete points and $u_{i}=u\left(\boldsymbol{g}\left(\boldsymbol{x}_{i}\right)\right.$ ) (and similarly for $v$ ). It can be checked that assuming $\psi_{1}$ to be the constant polynomial, then we have $w_{i}=\sqrt{2}\left(V^{-1}\right)_{1 i}$. Such a result is unfortunately minimal, i.e., only exact for integrands in $\mathcal{P}_{N}(T)$. However, the TSEM-1 leads to a diagonal mass matrix, the nice property shown by the SEM.

TSEM-2: In [19] the approach is similar except that both the electrostatic points introduced in [8] and the Fekete points are used. Moreover, a much more accurate integration rule is introduced. More precisely, if $g$ is a linear mapping, the Jacobian determinant $J$ is constant and in this case one has $I \approx \underline{u}^{t} W \underline{v}$, where $W=J V^{-t} V^{-1}$, with the superscript ${ }^{t}$ for "transposition" and where $\underline{u}$ and $\underline{v}$ gather the $u_{i}$ and $v_{i}, 1 \leqslant i \leqslant n$, respectively. Such an integration rule is exact if $u \circ g$ and $v \circ g$ are polynomials of $\mathcal{P}_{N}(T)$, which is by far superior to the TSEM-1. However, for the TSEM-2 the mass matrix is no-longer diagonal: being $u$ and $v$ the Lagrange polynomials built on the Fekete points, the elemental mass matrix equals $W$.

Extensions of this approach to the case where $g$ is no-longer a linear mapping are proposed in [10, 19]. In [19], the approach proposed in [11] is used for curved elements, whereas in [10] a more accurate computation of the matrix $W$ is proposed. However, in this latter case the matrix $W$ is no-longer proportional to the one of the reference triangle but depends on the shape of the triangular spectral element. In [10] it is suggested to store the matrix $W$ for each curved triangle. This requires rapidly a huge amount of memory space, even if as outlined in [10] curved triangles are not necessary for interior spectral elements. To avoid this severe drawback, we check here a simpler approach: following what is done for the usual SEM and in order to preserve the symmetry of the bilinear form, we use for $W$ the matrix $W=J^{1 / 2} V^{-t} V^{-1} J^{1 / 2}$, where $J$ is no-longer a scalar but the diagonal matrix $J=\operatorname{diag}\left\{J_{1}, \ldots, J_{n}\right\}$, with $J_{i}$ the Jacobian determinant at point $\boldsymbol{x}_{i}$. This simplified approach is used in next section to obtain the numerical results for the TSEM-2. 
TSEM-3: Let us introduce the set of $m$ quadrature points, say $\left\{\boldsymbol{y}_{i}\right\}_{i=1}^{m}$, allowing for an exact integration of polynomials, e.g., in $\mathcal{P}_{2 N}(T)$, using an integration rule as for the TSEM-1. Given the values at the approximation points of a polynomial $u_{N} \in \mathcal{P}_{N}(T)$, one can set up an interpolation matrix and differentiation matrices to compute, at the quadrature points, the values of $u_{N}$ and of its derivatives, respectively. In details, let $u_{N} \in \mathcal{P}_{N}(T)$. Knowing the $u_{i}=u_{N}\left(\boldsymbol{x}_{i}\right), 1 \leqslant i \leqslant n$, one can easily compute the $u_{N}\left(\boldsymbol{y}_{i}\right)$, $1 \leqslant i \leqslant m$. To this end we use the KD polynomials and, denoting by $\hat{u}_{j}$ the components of $u_{N}$ in the KD basis, we have $u_{i}=\sum_{j=1}^{n} \hat{u}_{j} \psi_{j}\left(\boldsymbol{x}_{i}\right)=$ $\sum_{j=1}^{n} V_{i j} \hat{u}_{j}$. In matrix form, with $\underline{\hat{u}}$ the vector of the $\hat{u}_{j}$, we have $\underline{u}=$ $V \underline{\hat{u}}$. Similarly, with $\underline{u}^{\prime}$ for the vector gathering the $u\left(y_{i}\right)$ and $V_{i j}^{\prime}=\psi_{j}$ $\left(\boldsymbol{y}_{i}\right)$, we obtain $\underline{u}^{\prime}=V^{\prime} \underline{\hat{u}}=V^{\prime} V^{-1} \underline{u}$. To compute derivatives, e.g., with respect to $r$, at the quadrature points we use the KD polynomials: if $\psi_{j}(\boldsymbol{x})=\sum_{k=1}^{n} \psi_{j}\left(\boldsymbol{x}_{k}\right) \phi_{k}(\boldsymbol{x})$, then $\partial_{r} \psi_{j}\left(\boldsymbol{y}_{i}\right)=\sum_{k=1}^{n} \psi_{j}\left(\boldsymbol{x}_{k}\right) \partial_{r} \phi_{k}\left(\boldsymbol{y}_{i}\right)$, so that we obtain for the differentiation matrix: $D^{\prime r}=V^{\prime r} V^{-1}$ with $\left(V^{\prime r}\right)_{i j}=$ $\partial_{r} \psi_{j}\left(\boldsymbol{y}_{i}\right)$.

Once knowing such differentiation matrices, $D^{\prime r}$ and $D^{\prime s}$, it is an easy task, by applying the chain rule, to compute derivatives at the quadrature points from the values at the approximation points. Note that if $\boldsymbol{g}$ is a non-linear mapping, such an approach is not equivalent to the computation of the derivatives at the Fekete points followed by an interpolation at the Gauss points. This latter approach would be more expensive and moreover not accurate enough to preserve the exponential convergence property.

The present approach, TSEM-3, shows some nice properties with respect to the TSEM-2, (i) in terms of computational time, to set up the stiffness matrix, (ii) because it does not require large memory space storages for curved triangles and finally, (iii) because it is very flexible, allowing, e.g., to enforce an exact integration only for integrands in $\mathcal{P}_{2 N-1}$, similarly to the SEM, in order to still decrease the computational time. The TSEM-3 requires of course the use of highly accurate integration rules based on Gauss points, as proposed in $[5,14,18]$. Unfortunately, in practice such integration rules are not yet known (or accessible) for large values of $N$. In this case it remains possible to use Gauss points based integration rules for the quadrangle, then using a mapping, say $\boldsymbol{h}: Q \rightarrow T$, from the reference quadrangle $Q=\{(r, s),-1 \leqslant r, s \leqslant 1\}$ to the reference triangle $T$. Such a mapping is, e.g., detailed for both $2 \mathrm{D}$ and $3 \mathrm{D}$ problems in [11], where it is moreover suggested to use the quadrature points and weights associated to the tensorial product of Jacobi polynomials $P_{i}^{1,0}$ and $P_{j}^{0,0}$ (Legendre polynomials), $0 \leqslant i, j \leqslant N_{Q}$. Such polynomials show indeed the property of being orthogonal with a weight proportional 
to the Jacobian determinant of $\boldsymbol{h}$ (2D case). With such an integration rule, the quadrature points are no-longer symmetric and their number is maximal: $\left(N_{Q}+1\right)^{2}$ points are required for an exact integration of polynomials over $T$ of degree $M=2 N_{Q}+1$ in each variable. In Fig. 1, we compare the distribution in $T$ of the Gauss points, issued from [6], with those derived from the Gauss points of the quadrangle. One should remark that in the former case some Gauss points are out of the triangle, which is acceptable for the quadrature points (but of coarse not for the approximation points) and in the latter that an a priori useless accumulation of points occurs in the upper vertex. In Table I we compare the number of quadrature points for each integration rule.

TSEM-4: Just as the TSEM-2, the TSEM-3 suffers from the fact that the mass matrix is not diagonal. This may constitute a drawback to solve, e.g., evolution problems with an explicit scheme. To recover a diagonal mass matrix one may think to use the mass-lumping technique. Such an approach resumes to use for the mass matrix an integration rule such that the matrix turns out to be diagonal. For $\mathcal{P}_{1}$-finite elements, the mass lumping technique is equivalent to the use of an integration rule only based on the vertices of the triangle. In the framework of the TSEM, applying the same idea suggests to use the integration rule based on
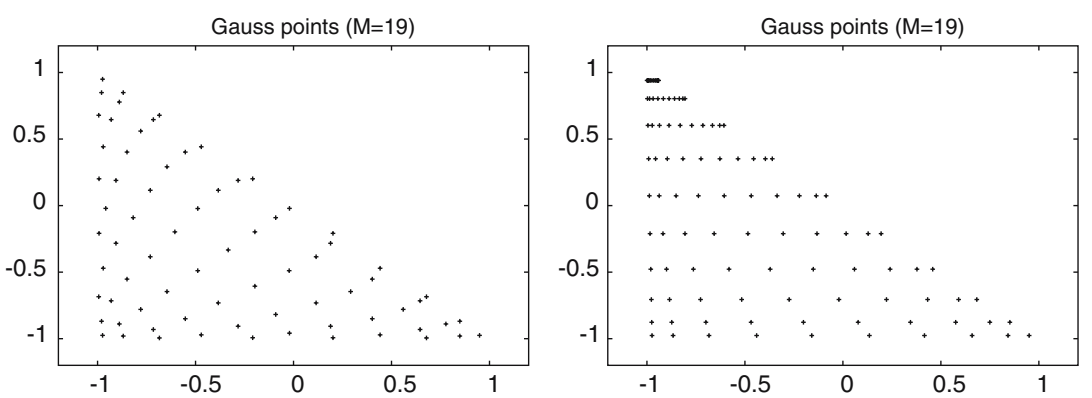

Fig. 1. Quadrature points in the reference triangle for $M=19$ with a symmetric (left) and non-symmetric (right) distribution.

Table I. Number of Quadrature Points for Different Values of $M$ and for each Integration Rule

\begin{tabular}{lccccccr}
\hline Integration rule/M & 7 & 9 & 11 & 13 & 15 & 17 & 19 \\
\hline Sym. & 13 & 19 & 27 & 37 & 48 & 61 & 73 \\
Non-sym. & 16 & 25 & 36 & 49 & 64 & 81 & 100 \\
\hline
\end{tabular}


Fekete points to set up the mass matrix and on the Gauss points for the stiffness matrix and for the source term, i.e., a compromise between the TSEM-1 and TSEM-3.

\section{NUMERICAL RESULTS}

Comparisons are carried out for the following elliptic problem:

$$
\begin{aligned}
& -\nabla^{2} u+u=f \quad \text { in } \quad \Omega \\
& u=u_{D} \quad \text { on } \Gamma_{D} \subset \partial \Omega, \quad \partial_{n} u=0 \quad \text { on } \quad \Gamma_{N}=\partial \Omega \backslash \Gamma_{D}
\end{aligned}
$$

with $\partial_{n}$ for the normal derivative, $\Omega$ for the computational domain and where $f, u_{\Gamma}$, are smooth functions defined on $\Omega, \Gamma_{D}$, respectively.

\subsection{Elemental Tests}

We consider for $\Omega$ only one spectral element and compare the different TSEM. Comparisons are also provided with a collocation method and, considering a quadrilateral element rather than a triangular one, with the usual SEM. For the collocation method no integration rule is required since it is the strong form of the partial differential equation which is solved.

Computations have been carried out for problem (1) with Dirichlet boundary conditions $\left(\Gamma_{N}=\emptyset\right)$ and source term given in such a way that the exact solution reads: $u_{e x}=\sin (2 x+y) \sin (x+1) \sin (1-y)$. The computational domains are deduced from the reference triangle $T$ (or reference quadrangle $Q$ ) by the mapping: $x=r+0.5(s+1)+\epsilon\left(r^{3}+s^{2}\right), \quad y=$ $0.5 s+\epsilon\left(r^{2}+s^{3}\right)$, where $\epsilon=0$ or $\epsilon=0.1$. In the former case the mapping is linear, so that there is only a stretching of the reference triangle whereas in the latter we have a deformation. In Fig. 2 are shown the Fekete points $(N=18)$ for the triangle mapped with $\epsilon=0$ and $\epsilon=0.1$.

In semi-log representation, the variations of the max-norm of the error at the Fekete points obtained with the linear mapping $(\epsilon=0)$ are shown in Fig. 3 (left). For the TSEM-3 and TSEM-4, computations have been made using the mapping $\boldsymbol{h}: Q \rightarrow T$. We do not have indeed the Gauss quadrature points and weights of the triangle for $M>19$, which limits $N \lesssim 10$. Essentially it appears that the exponential rate of convergence is achieved as soon as the integration rule is accurate enough. Thus, the TSEM- 1 and TSEM-4 do not yield a satisfactory result, in contrast to the TSEM-2 and TSEM-3. However, as expected, the computational time was longer for the TSEM-2. Also one observes that both the collocation method and the usual SEM, with a rectangular element, yield the expected exponential rate of convergence. 
Results obtained with the non-linear mapping $(\epsilon=0.1)$ are shown in Fig. 3 (right). Here, the only satisfactory results are obtained with the TSEM-3 and, of course, with the collocation method and the usual SEM. For the TSEM-2, one has initially the expected decrease of the error before saturation. Thus, the simplified form of matrix $W$ does not allow to obtain spectrally accurate results with curved elements.

As mentioned in the previous section, the TSEM-3 is very flexible in the sense that one can decrease the computational time by decreasing the polynomial maximum degree $M$, for which the Gauss integration is exact. Figure 4 (left) shows results obtained for $N_{Q}=\{N, N-1, N-2, N-3\}$ and also when using the symmetric quadrature rule with $M=19$. Essentially, in the former case one observes satisfactory convergence rates and even similar errors, except for $N_{Q}=N-3$, whereas in the latter case results are not satisfactory for $N=15$ and $N=18$, due to a not enough accurate evaluation of the integrals. Note however that such results may depend on the degree of the polynomial mapping (here is 3 for $\epsilon=0.1$ ).
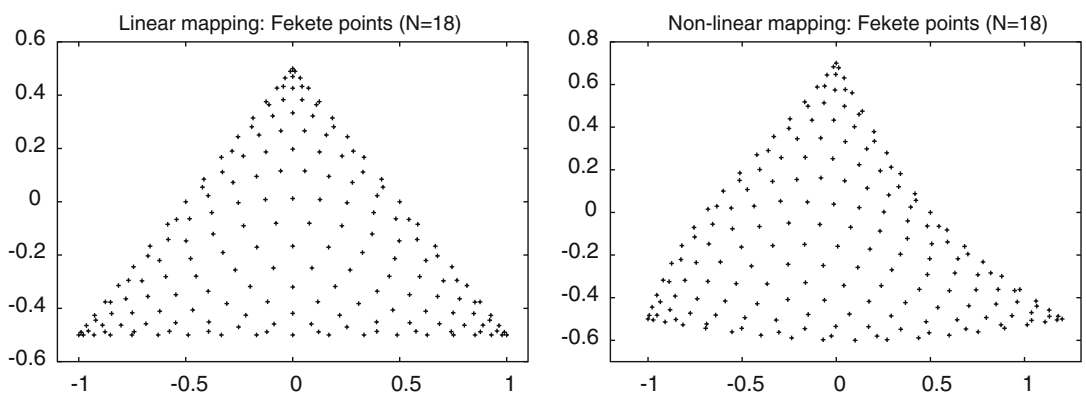

Fig. 2. Approximation points for $\epsilon=0$ (left) and $\epsilon=0.1$ (right), $N=18$.
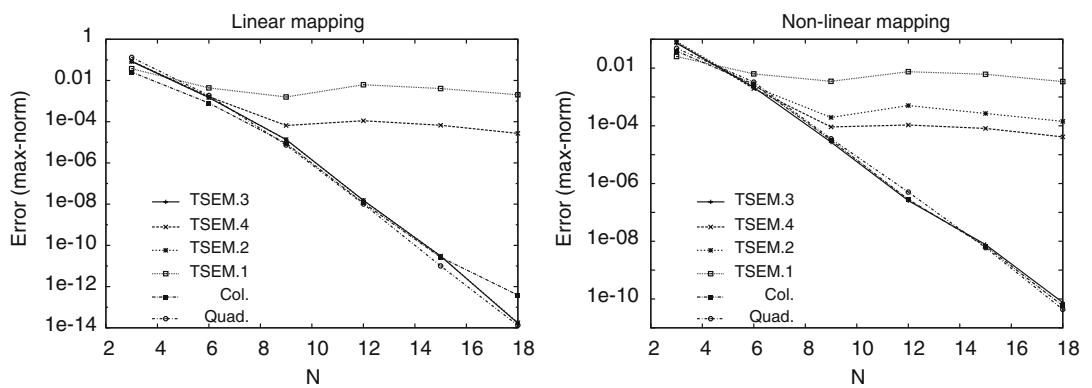

Fig. 3. $N$-convergence study for different spectral approximations with linear (left) and non-linear mapping (right), $N=18$. 

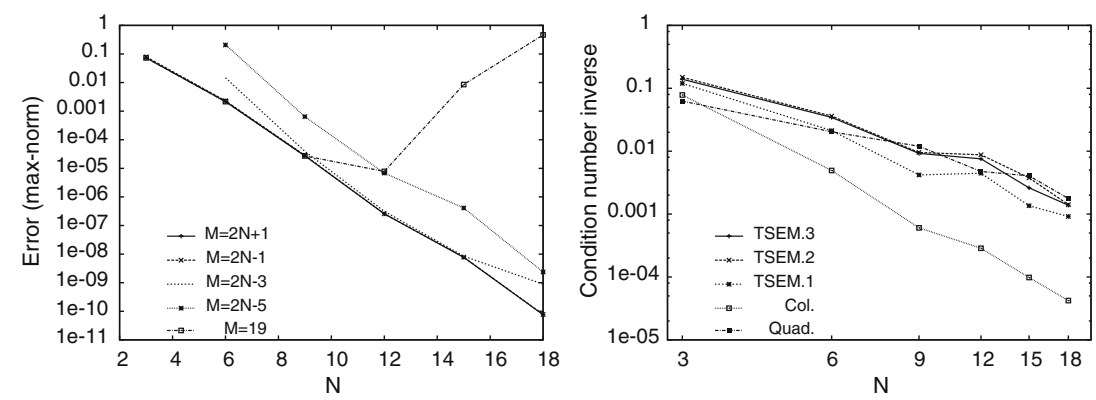

Fig. 4. TSEM-3: $N$-convergence study for several values of $M$ with non-linear mapping (left) and inverse of the condition number for different spectral approximations (right).

One may ask why using a TSEM when the collocation method appears to yield very satisfactory results. However: (i) the study has been carried out for only one element. When a set of elements is considered then the transmission condition is the $C^{1}$ continuity of the solution, which may be difficult to implement; (ii) better condition numbers are obtained with the TSEM. This is visualized in Fig. 4 (right) which shows the variations of the inverse of the condition number of the final matrix (as computed with the routine DGESVX of the LAPACK library). The TSEM-3 condition number follows the one of the TSEM-2. This could be expected since the tiny difference that can be observed only results from the use of a non-linear mapping (final matrices are identical with a linear mapping).

\subsection{TSEM-3: A $N$ - and $h$-Convergence Study}

Here we check the convergence properties of the TSEM-3 with respect to $N$ and to the number of spectral elements. To this end we consider again the problem (1), with now $\Omega=\left\{(x, y), \quad 0 \leqslant x \leqslant 1, \quad 0 \leqslant y \leqslant 1-x^{2}\right\}$ and $\Gamma_{D}=\left\{\left(x, 1-x^{2}\right), 0 \leqslant x \leqslant 1\right\}$. To avoid reaching the machine accuracy, we have considered problem (1) with $f$ and $u_{D}$ consistent with the stiff function $u=\tanh 10\left(x^{2}+y^{2}-0.5^{2}\right)$.

Figure 5 (left) shows the approximation points for $N=9$ of a 42 triangle macro-mesh. In the following, computations are done with macro-meshes of $N_{e l}=21,42,64$, and 90 triangles and the polynomial approximation degree in each triangle is chosen among the values $N=$ $3,6,9,12,15,18$. A quadratic transformation is considered for each triangle touching the curved boundary in order to describe it perfectly. This can be done easily, by using the Lagrange polynomials involved in classical $\mathcal{P}_{2}$-finite element approximations. 

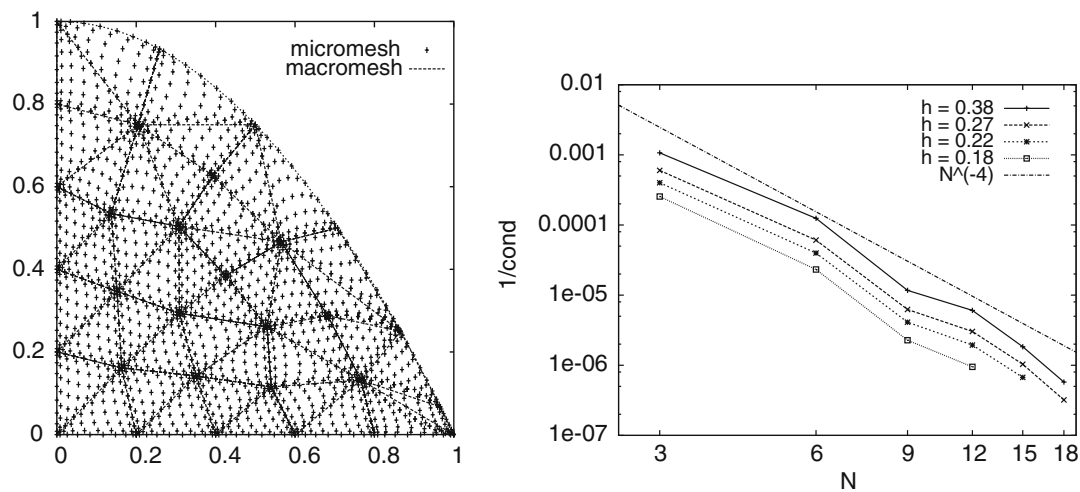

Fig. 5. Approximation points for $N=9$ on the macro-mesh of 42 triangles (left) and inverse of the condition number of the problem matrix (right).

Figure 5 (right) shows the log-plot of the inverse of the condition number versus the adopted degree $N$ for the TSEM-3. As discussed in [12], the condition number shows a $O\left(N^{4}\right)$ behavior, in agreement with what is usually obtained for $h p$-finite element methods ( $h$ : element mesh size, $p$ : polynomial approximation degree).

Figure 6 (left) shows the log-plots of the error for different choices of $N$. As expected the convergence to the exact solution is of algebraic type and achieved with an order of accuracy close to $(N+1)$ with respect to $h$. The value $N=3$ is too small to allow for a correct approximation. Figure 6 (right) shows the semi log-plots of the error for the considered choices of $h$. As expected exponential convergence is achieved with respect to $N$.
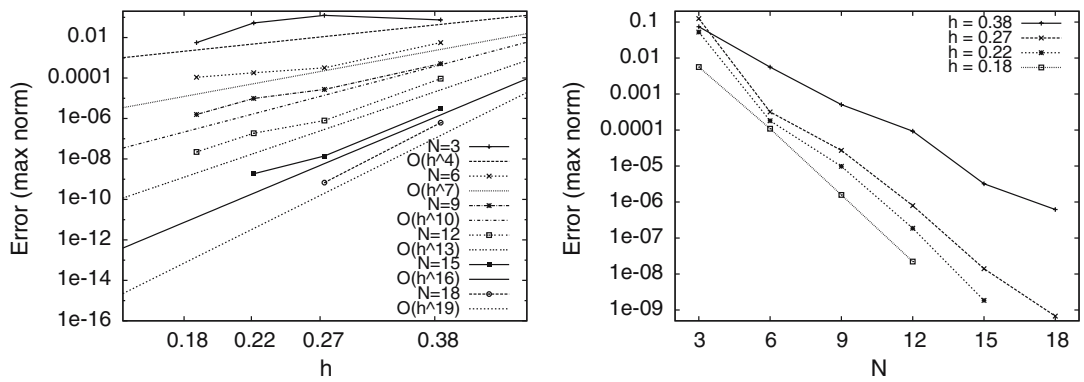

Fig. 6. A $h$-convergence (left) and $N$-convergence (right) study for the TSEM-3 solution of problem (1) for different values of $N, h$, respectively. 


\section{CONCLUSION}

In comparison with approaches previously proposed [10, 15, 19], the TSEM that we have described (TSEM-3) makes use of two sets of points: the approximation points and the quadrature points. This seems in fact the only possible strategy, at least for elliptic problems, as also advocated in [17]. For the approximation points we have chosen Fekete points and for quadrature points we suggest to use Gauss points for simplices. However, for the quadrature points we have also used the Gauss points associated to the quadrangle. The efficiency of the TSEM-3 results from the introduction of differentiation matrices allowing to compute the derivatives at the quadrature points from values given at the approximation points, which is not usual in the frame of spectral methods. Moreover, there is the possibility of "sub-integrating" by using, e.g., an integration rule only exact in $\mathcal{P}_{2 N-3}$ rather than in $\mathcal{P}_{2 N}$ thus yielding differentiation matrices of smaller size.

\section{REFERENCES}

1. Bos, L. (1991). On certain configurations of points in $\mathbb{R}^{n}$ which are unisolvent for polynomial interpolation. J. Approx. Theory 64, 271-280.

2. Bos, L., Taylor, M. A., and Wingate, B. A. (2001). Tensor product Gauss-Lobatto points are Fekete points for the cube. Math. Comp. 70, 1543-1547.

3. Chen, Q., and Babuška, I. (1995). Approximate optimal points for polynomial interpolation of real functions in an interval and in a triangle. Comput. Methods Appl. Mech. Eng. 128, 485-494.

4. Chen, Q., and Babuška, I. (1996). The optimal symmetrical points for polynomial interpolation of real functions in a tetrahedron. Comput. Methods Appl. Mech. Eng. 137, 8994.

5. Cools, R. (2002). Advances in multidimensional integration. J. Comput. Appl. Math. 149, $1-12$.

6. Demkowicz, L., Walsh, T., Gerdes, K., and Bajer, A. (1998). 2D hp-adaptative finite element package Fortran 90 implementation (2Dhp90), TICAM Report 98-14.

7. Dubiner, M. (1991). Spectral methods on triangles and other domains. J. Sci. Comput. 6, 345-390.

8. Hesthaven, J. S. (1998). From electrostatic to almost optimal nodal sets for polynomial interpolation in a simplex. SIAM J. Numer. Anal. 35, 655-676.

9. Hesthaven, J. S., and Teng, C. H. (2000). Stable spectral methods on tetrahedral elements. SIAM J. Sci. Comput. 21, 2352-2380.

10. Hesthaven, J. S., and Warburton, T. (2002). Nodal high-order methods on unstructured grids. J. Comput. Phys. 181: 186-221.

11. Karniadakis, G. E., and Sherwin, S. J. (1999). Spectral hp Element Methods for CFD, Oxford University Press, London.

12. Pasquetti, R., and Rapetti, F. (2004). Spectral element methods on triangles and quadrilaterals: comparisons and applications. J. Comput. Phys. 198, 349-362.

13. Stroud, A. H., and Secrest, D. (1966). Gaussian Quadrature Formulas, Prentice Hall, NJ.

14. Stroud, A. H. (1971). Approximate Calculations of Multiple Integrals, Prentice Hall, NJ. 
15. Taylor, M. A., Wingate, B. A., and Vincent, R. E. (2000). An algorithm for computing Fekete points in the triangle. SIAM J. Numer. Anal. 38, 1707-1720.

16. Taylor, M. A., and Wingate, B. A. (2000). A generalized diagonal mass matrix spectral element method for non-quadrilateral elements. Appl. Num. Math. 33, 259-265.

17. Taylor, M. A., Wingate, B. A., and Bos, L. P. (2004). A new algorithm for computing Gauss-like quadrature points, ICOSAHOM 2004 proc., Brown University.

18. Wandzura, S., and Xiao, H. (2003). Symmetric quadrature rules on a triangle. Comput. Math. Appl. 45, 1829-1840.

19. Warburton, T., Pavarino, L., and Hesthaven, J. S. (2000). A pseudo-spectral scheme for the incompressible Navier-Stokes equations using unstructured nodal elements. J. Comput. Phys. 164, 1-21. 